\title{
Diagnostic Value of MRI for Detecting Recurrent Soft-Tissue Sarcoma in a Long-Term Analysis at a Multidisciplinary Sarcoma Center
}

\section{Sam Sedaghat ( $\sim$ samsedaghat1@gmail.com )}

University Hospital Schleswig-Holstein - Campus Kiel: Universitatsklinikum Schleswig-Holstein https://orcid.org/0000-0003-2804-3718

\section{Maya Sedaghat}

University Hospital Schleswig-Holstein - Campus Kiel: Universitatsklinikum Schleswig-Holstein Jens Meschede

Klinikum Dortmund gGmbH

\section{Olav Jansen}

University Hospital Schleswig-Holstein - Campus Kiel: Universitatsklinikum Schleswig-Holstein

\section{Marcus Both}

University Hospital Schleswig-Holstein - Campus Kiel: Universitatsklinikum Schleswig-Holstein

\section{Research article}

Keywords: diagnostic accuracy, MRI, soft-tissue sarcoma, long-term analysis, sarcoma center

Posted Date: November 20th, 2020

DOl: https://doi.org/10.21203/rs.3.rs-111370/v1

License: (c) (1) This work is licensed under a Creative Commons Attribution 4.0 International License. Read Full License

Version of Record: A version of this preprint was published at BMC Cancer on April 13th, 2021. See the published version at https://doi.org/10.1186/s12885-021-08113-y. 


\section{Abstract}

Background: To assess the long-term diagnostic accuracy of MRI for detecting recurrent soft-tissue sarcoma at a multidisciplinary sarcoma center.

Methods: In all, 1055 postoperative follow-up MRIs of 204 patients were included in the study. MRI followup scans were systematically reviewed for true-positive/-negative and false-positive/-negative results in detecting recurrent tumor. Available pathological reports and follow-up MRIs were set as reference.

Results: The median age of the patients was $55.3 \pm 18.2$ years. Of the patients, $34.8 \%$ presented with recurrences. Here, 65 follow-up scans were true positive, 23 false positive, 6 false negative, and 961 true negative. The overall sensitivity and specificity of MRI for detecting recurrences were $92 \%$ and $98 \%$, respectively, with an accuracy of $97 \%$. For intramuscular lesions and after surgery alone the sensitivity was higher (95\% and $97 \%$ ) than for subcutaneous lesions and surgery with additional radiation therapy ( $83 \%$ and $86 \%$, respectively), at similarly high specificities (96-98\%). The 6 false-negative results were found in streaky $(n=2)$ and small ovoid/nodular $(n=4)$ recurring lesions. The false-positive lesions imitated streaky $(n=14)$, ovoid/nodular $(n=8)$, and polycyclic/multilobulated recurring tumors $(n=1)$. All false-positive results were found in patients in whom the primary tumors were polycyclic/multilobulated in appearance.

Conclusion: MRI shows a high diagnostic accuracy for detecting recurrent STS, with a high sensitivity and specificity. The diagnostic accuracy decreases in subcutaneous lesions and after surgery with radiation therapy, compared to intramuscular lesions and surgery alone. Radiologists should pay particular attention to streaky and small ovoid/nodular recurring lesions and patients with polycyclic/multilobulated primary tumors.

\section{Background}

Soft-tissue sarcomas (STS) constitute a rare and heterogeneous group of tumors, accounting for only $1 \%$ of adult malignancies [1]. Due to the rarity of these malignancies, there is often only little experience in dealing with STS and in postoperative surveillance outside of specialty centers [2]. More than 50 different subtypes have been described, and the extremities are the most common sites of STS [1,3]. Surgery is the most common treatment option for soft-tissue sarcomas with additional radiotherapy in selected cases [4]. In the literature, different strategies for postoperative surveillance of STS patients have been reported. Nevertheless, a unified strategy is still lacking [1,5-7]. The most commonly used imaging modalities for postoperative follow-up are ultrasound and magnetic resonance imaging (MRI) [8]. MRI is the preferred imaging modality for postoperative follow-up in STS patients and, consequently, for detecting local recurrences. The diagnostic value of MRI has already been investigated for numerous tumors, such as brain metastasis or other malignancies [9-11]. Nevertheless, current studies on the diagnostic value of MRI in the postoperative surveillance of STS patients are rare and mostly include only a few cases of STS [12-14]. Therefore, we analyzed the diagnostic value of MRI for detecting recurrent 
STS in the long-term, postoperative follow-up at a multidisciplinary sarcoma center. Furthermore, we analyzed whether the localization of STS in the soft tissue or the type of therapy has an impact on the diagnostic value of MRI.

\section{Methods}

Patients: A total of 1707 postoperative follow-up MRI scans were performed in 242 patients with histologically proven STS. Thirty-eight patients were excluded due to insufficient imaging and pathological data. Ultimately, 204 patients with a total of 1286 postoperative follow-up MRI scans were included in our study and presented complete data on imaging. For statistical purposes, the last examination of each patient and those examinations in which proof of predictive values could not be determined due to incomplete reference follow-ups were excluded $(n=231)$. Either core needle or open biopsy was performed in all lesions suspected of being recurrences $(n=88)$. In these patients, the pathological reports were set as reference and the radiological findings were correlated to the pathological reports. All other MRIs were viewed during the subsequent MRI follow-up examinations, showing whether lesions had been overlooked in the previous MRIs. These subsequent MRI follow-ups took place after 3 to 6 months. The follow-up MRIs were reviewed by two dedicated musculoskeletal radiologists with a minimum of five years of experience in sarcoma diagnostics, with findings reached by consensus. The reports were divided into two groups: presence of recurrence and absence of recurrence. From these findings we extracted true-positive/-negative and false-positive/-negative MRI findings in detecting recurrent STS.

Magnetic Resonance Imaging: All patients were examined with a 1.5-Tesla MRI system (MAGNETOM Symphony, Siemens Healthineers, Erlangen, Germany). The MRI protocol included the following pulse sequences: T2-weighted (T2w) TSE (TE: 64-114 ms, TR: 3010-5840 ms, FOV: 22-44 cm²), T1-weighted (T1w) SE (TE: 10-14 ms, TR: 587-868 ms, FOV: 22-44 cm²), proton density-weighted (PDw) FS (TE: 2636 ms, TR: $2740-4610$ ms, FOV: 22-40 cm²), or Turbo-Inversion Recovery Magnitude (TIRM) (TE: 6877 ms, TR: 4410-6980 ms, FOV: 37-45 cm²) and contrast-enhanced T1w SE FS (10-13 ms, TR: 533$1440 \mathrm{~ms}$, FOV: $22-45 \mathrm{~cm}^{2}$ ). Slice thickness was $4-6 \mathrm{~mm}$.

Statistical data: Diagnostic accuracy was determined by calculating predictive values (positive and negative), sensitivity, specificity, and accuracy using Fisher's exact test and $2 \times 2$ tables. The $95 \%$ confidence interval was determined using the Wald test. Statistical significance for all tests was set at a level of $p<0.05$. Statistical analysis was done using the IBM-SPSS version 26.0 software package (IBM, Armonk, NY, USA).

\section{Results}

The median age of the patients was 55.3 years (Min.: 10, Max.: 88, SD: 18.2). Of the patients, $52.9 \%$ were male $(n=108)$. The median recurrence-free follow-up interval on MRI was 39 months (Min.: 3, Max.: 161, SD: 29.9$)$. Here, $34.8 \%$ of the patients presented with recurrences. Sixty-five follow-up MRI scans were 
diagnosed as true positive, 23 as false positive, 6 as false negative, and 961 as true negative (Table 1 and Fig. 1). Overall, sensitivity and specificity of MRI for detecting recurrences were 0.92 and 0.98 , respectively, with an accuracy of 0.97 (Table 2). For intramuscular lesions the sensitivity was higher than for subcutaneous lesions ( 0.95 and 0.83 , respectively), at similarly high specificities ( 0.97 and 0.98 , respectively; Tables 3 and 4). Furthermore, the sensitivity was higher in patients after surgery alone than after surgery with additional radiation therapy ( 0.97 and 0.86 , respectively), at similar specificities $(0.96$ and 0.98 , respectively; Tables 3 and 5). The 6 false-negative results were found in streaky $(n=2)$ and small ovoid/nodular $(n=4)$ recurrences. The false-positive lesions imitated streaky $(n=14)$, ovoid/nodular ( $n=8$; Fig. 2), and polycyclic/multilobulated recurring lesions $(n=1)$. All false-positive results were found in patients in whom the primary STS was polycyclic/multilobulated in appearance. Furthermore, 22 of the 23 false-positive results were derived from patients with R0 resection (95.7\%). Altogether, $74.5 \%$ of the patients underwent $\mathrm{R} 0$ resection.

\section{Discussion}

In our study we investigated the diagnostic accuracy of standard MRI for detecting recurring soft-tissue sarcoma at a multidisciplinary sarcoma center. Soft-tissue tumors include benign and malignant tumors and tumor-like lesions [15]. In our study we dealt with malignant lesions only, so-called soft-tissue sarcomas (STS). STS constitute a rare and heterogeneous group of tumors, which account for only about $1 \%$ of all malignancies [1]. The most recent histological classification of STS comes from the current WHO histological typing, in which more than 50 subtypes are described $[1,15,16]$. Previous studies on soft-tissue sarcoma recurrence have reported a wide range of recurrence rates of up to $50 \%[17,18]$. Due to the rarity of STS, imaging studies on STS still remain scarce. Furthermore, most previous studies dealt with imaging features rather than with the diagnostic accuracy. Additionally, previous diagnostic studies mostly included small patient numbers or reviewed the available literature. For postoperative surveillance, MRI is the imaging modality of choice and is widely used to assess STS recurrence $[14,19]$, as MRI has the advantage of a high soft-tissue contrast and no radiation. Nevertheless, differentiation between posttreatment changes and recurrent STS on MRI is often reported to be challenging [20]. Previous publications have often shown that post-treatment changes and scar tissue can obscure recurring STS, which leads to unnecessary biopsies [21]. In patients with local recurrences combined treatment with surgery and additional radiation therapy is usually chosen to improve local control $[1,22]$. Nevertheless, a decision regarding the use of additional radiation therapy should be evaluated from case to case $[1,23]$. Before starting the therapy, core needle biopsy is often performed to identify the pathology of the suspected lesions [24]. To start therapy quickly and to avoid unnecessary biopsy, precise post-operative MRI diagnostics are indispensable. Therefore, according to our study, it is of high clinical and diagnostic importance to determine the diagnostic reliability of MRI for postoperative surveillance of sarcoma patients in a real-world cohort over a long time period. In our study, both sensitivity (92\%) and specificity $(98 \%)$ were high overall, even after both surgery and radiation therapy and in both subcutaneous and intramuscular lesions. Indeed, the sensitivity was lower after radiation therapy than after surgery alone and in subcutaneous lesions, compared to intramuscular lesions, but the sensitivity still remained at a 
high level. Reasons for the decreased sensitivities could lie in the increased rate of soft-tissue alterations after additional radiation therapy[21,25] and the usually smaller sizes of the subcutaneous lesions. These two findings ultimately lead to more difficulty in distinguishing between post-treatment changes and recurring tumor. The range of the described sensitivity and specificity is high. However, some previous publications reported a lower specificity, ultimately leading to unneeded biopsies [14, 21, 26]. Afonso et al. described a sensitivity and specificity of only $58 \%$ and $73 \%$, respectively, for conventional MRI [19], while Del Grande F. et al. assessed a sensitivity and specificity of MRI of $100 \%$ and $52 \%$, respectively, in detecting tumor recurrence in nonenhanced $\mathrm{MRI}$, and $100 \%$ and $97 \%$, respectively, in contrast-enhanced MRI [20]. In a recent review, Pennington A. et al. calculated a mean sensitivity and specificity of $88 \%$ and $86 \%$, respectively, for local recurrences of primary vertebral tumors[27]. Other authors showed sensitivities and specificities of $64-88 \%$ and $85-96 \%$, respectively, for MRI for detecting soft-tissue tumors [19, 28-31]. Nevertheless, none of the previous studies investigated how MRI performed in the real-world clinical setting. Previous publications reported a lack of specificity of MRI for detecting recurring STS in nonenhanced T1- and T2-weighted images [21, 32, 33]. Therefore, contrastenhanced MRI is reported to improve the diagnostic accuracy of MRI [34].

In our study, all of the patients were examined using contrast-enhanced MRI. Our data emphasize that MRI is a highly valuable imaging modality in the long-term postoperative surveillance of STS patients. Nevertheless, we found 23 false-positive and 6 false-negative results (8.5\%). These 6 cases were all derived from streaky and small ovoid/nodular lesions, which were difficult to distinguish from the surrounding post-treatment tissue. A recent study showed that distinct post-therapeutic changes are the main reason for false-negative results on MRI [35]. All false-positive results were found in patients in whom the primary STS was polycyclic/multilobulated. This may well be due to the fact that polycyclic/multilobulated primary tumors are larger than other configured STS in the mean and therefore perhaps lead to more heterogeneous post-treatment tissue changes. Furthermore, 22 of the 23 falsepositive results were derived from patients with $\mathrm{RO}$ resection. This is in contrast to a recent study showing that microscopic positive margins were the main reason for false-positive results [35].

Therefore, radiologists should pay particular attention to patients in whom the primary STS was polycyclic/multilobulated in shape and should carefully screen the soft tissue for streaky or small ovoid/nodular recurrences. However, this fact also demonstrates the limitations of MRI surveillance. In cases of recurrent lesions with a a grainy appearance, it may become difficult to correctly detect a recurring tumor in the surrounding tissue. Therefore, in suspected or unclear cases, the subsequent MRI follow-up examinations should take place after 3 to 6 months.

Our study has some limitations, as it is a single-center study with a retrospective design. Nevertheless, we could include 204 patients in a 12-year survey with a total of $1055 \mathrm{MRI}$ follow-up scans. Another limitation is the verification of the true-positive/-negative and false-positive/-negative results. Biopsy was only performed in patients in whom recurrence was suspected. In the other cases, radiologists evaluated whether recurrences were overlooked or not. Therefore, we cannot completely rule out that individual findings might be false. 


\section{Conclusion}

MRI shows a high diagnostic accuracy for detecting recurring STS in the long term, with a high sensitivity (92\%) and specificity (98\%). After resection of subcutaneous primary tumors and after radiation therapy, the sensitivity decreases to $83 \%$ and $86 \%$, respectively, compared to intramuscular lesions and surgery alone (95\% and $97 \%$, respectively). Radiologists should pay particular attention to patients in whom the primary tumor was polycyclic/multilobulated in appearance and should carefully screen the posttreatment soft tissue for streaky and small ovoid/nodular recurrences, which are often difficult to distinguish from post-treatment changes.

\section{Abbreviations}

MRI - Magnetic resonance imaging

STS - soft-tissue sarcoma

\section{Declarations}

\section{Ethics approval and consent to participate}

This study was approved by the responsible ethics committee of the Ruhr-University Bochum, Germany. All patients gave their verbal informed consent before examination. Written informed consent was waived because of the retrospective nature of the study and the analysis of anonymous clinical data.

The consent was noted in a general patient informed consent sheet for all patients before examination.

\section{Consent to publish}

Not applicable.

\section{Availability of data and materials}

The datasets used and/or analysed during the current study are available from the corresponding author on reasonable request.

\section{Competing interests}

The authors declare that they have no competing interests.

\section{Funding}

Not applicable.

\section{Authors' contributions}


SS has made substantial contributions to the conception/design of the work, acquisition, analysis, interpretation of data and have drafted the work. SS has approved the submitted version (and any substantially modified version that involves the author's contribution to the study) and has agreed both to be personally accountable for the author's own contributions and to ensure that questions related to the accuracy or integrity of any part of the work, even ones in which the author was not personally involved, are appropriately investigated, resolved, and the resolution documented in the literature.

MS has made substantial contributions to the conception/design of the work, acquisition, analysis, interpretation of data and have drafted the work. MS has approved the submitted version (and any substantially modified version that involves the author's contribution to the study) and has agreed both to be personally accountable for the author's own contributions and to ensure that questions related to the accuracy or integrity of any part of the work, even ones in which the author was not personally involved, are appropriately investigated, resolved, and the resolution documented in the literature.

JM has made substantial contributions to the conception/design of the work, analysis, interpretation of data and substantively revised the draft. JM has approved the submitted version (and any substantially modified version that involves the author's contribution to the study) and has agreed both to be personally accountable for the author's own contributions and to ensure that questions related to the accuracy or integrity of any part of the work, even ones in which the author was not personally involved, are appropriately investigated, resolved, and the resolution documented in the literature.

OJ has made substantial contributions to the analysis and interpretation of data, and substantively revised the draft. OJ has approved the submitted version (and any substantially modified version that involves the author's contribution to the study) and has agreed both to be personally accountable for the author's own contributions and to ensure that questions related to the accuracy or integrity of any part of the work, even ones in which the author was not personally involved, are appropriately investigated, resolved, and the resolution documented in the literature.

MB has made substantial contributions to the analysis and interpretation of data, and substantively revised the draft. MB has approved the submitted version (and any substantially modified version that involves the author's contribution to the study) and has agreed both to be personally accountable for the author's own contributions and to ensure that questions related to the accuracy or integrity of any part of the work, even ones in which the author was not personally involved, are appropriately investigated, resolved, and the resolution documented in the literature.

\section{Acknowledgements}

Not applicable.

\section{References}


1. Mehren M von, Randall RL, Benjamin RS, Boles S, Bui MM, Ganjoo KN, et al. Soft Tissue Sarcoma, Version 2.2018, NCCN Clinical Practice Guidelines in Oncology. J Natl Compr Canc Netw. 2018;16:536-63. doi:10.6004/jnccn.2018.0025.

2. Ratan R, Patel SR. Chemotherapy for soft tissue sarcoma. Cancer. 2016;122:2952-60. doi:10.1002/cncr.30191.

3. Pazdur R, Coia LR, Hoskins WJ, Wagman LD. Cancer management: a multidisciplinary approach: F.A. Davis Company; 2003.

4. Clark MA, Fisher C, Judson I, Thomas JM. Soft-tissue sarcomas in adults. New England Journal of Medicine. 2005;353:701-11.

5. Kane III JM. Surveillance strategies for patients following surgical resection of soft tissue sarcomas. Current opinion in oncology. 2004;16:328-32.

6. Patel SA, Royce TJ, Barysauskas CM, Thornton KA, Raut CP, Baldini EH. Surveillance imaging patterns and outcomes following radiation therapy and radical resection for localized extremity and trunk soft tissue sarcoma. Annals of surgical oncology. 2017;24:1588-95.

7. Whooley BP, Gibbs JF, Mooney MM, McGrath BE, Kraybill WG. Primary extremity sarcoma: what is the appropriate follow-up? Annals of surgical oncology. 2000;7:9-14.

8. Briccoli A, Galletti S, Salone M, Morganti A, Pelotti P, Rocca M. Ultrasonography is superior to computed tomography and magnetic resonance imaging in determining superficial resection margins of malignant chest wall tumors. Journal of ultrasound in medicine. 2007;26:157-62.

9. Hosalkar HS, Garg S, Moroz L, Pollack A, Dormans JP. The diagnostic accuracy of MRI versus CT imaging for osteoid osteoma in children. Clin Orthop Relat Res. 2005:171-7. doi:10.1097/01.blo.0000151426.55933.be.

10. Schellinger PD, Meinck HM, Thron A. Diagnostic accuracy of MRI compared to CCT in patients with brain metastases. Journal of neuro-oncology. 1999;44:275-81.

11. Davies M, Cassar-Pullicino VN, Davies AM, McCall IW, Tyrrell PNM. The diagnostic accuracy of MR imaging in osteoid osteoma. Skeletal Radiol. 2002;31:559-69. doi:10.1007/s00256-002-0546-4.

12. Bolzoni A, Cappiello J, Piazza C, Peretti G, Maroldi R, Farina D, Nicolai P. Diagnostic accuracy of magnetic resonance imaging in the assessment of mandibular involvement in oral-oropharyngeal squamous cell carcinoma: a prospective study. Archives of Otolaryngology-Head \& Neck Surgery. 2004;130:837-43.

13. Kaste SC, Hill A, Conley L, Shidler TJ, Rao BN, Neel MM. Magnetic resonance imaging after incomplete resection of soft tissue sarcoma. Clin Orthop Relat Res. 2002;397:204-11.

14. Erfanian Y, Grueneisen J, Kirchner J, Wetter A, Podleska LE, Bauer S, et al. Integrated 18F-FDG $\mathrm{PET} / \mathrm{MRI}$ compared to MRI alone for identification of local recurrences of soft tissue sarcomas: a comparison trial. European journal of nuclear medicine and molecular imaging. 2017;44:1823-31.

15. Gielen JL, Schepper AM de, Vanhoenacker F, Parizel PM, Wang XL, Sciot R, Weyler J. Accuracy of MRI in characterization of soft tissue tumors and tumor-like lesions. A prospective study in 548 patients. European radiology. 2004;14:2320-30. 
16. Fletcher C. Pathology and genetics of tumors of soft tissue and bone. World Health Organization Classification of Tumors. 2002;4:35-46.

17. James SLJ, Am Davies. Post-operative imaging of soft tissue sarcomas. Cancer Imaging. 2008;8:8.

18. Kransdorf MJ, Murphey MD. Soft tissue tumors: post-treatment imaging. Radiologic Clinics. 2006;44:463-72.

19. Afonso PD, Kosinski AS, Spritzer CE. Following unenhanced MRI assessment for local recurrence after surgical resection of mesenchymal soft tissue tumors, do additional gadolinium-enhanced images change reader confidence or diagnosis? European journal of radiology. 2013;82:806-13.

20. Del Grande F, Subhawong T, Weber K, Aro M, Mugera C, Fayad LM. Detection of soft-tissue sarcoma recurrence: added value of functional MR imaging techniques at 3.0 T. Radiology. 2014;271:499511.

21. Garner HW, Kransdorf MJ, Bancroft LW, Peterson JJ, Berquist TH, Murphey MD. Benign and malignant soft-tissue tumors: posttreatment MR imaging. Radiographics. 2009;29:119-34.

22. Catton C, Davis A, Bell R, O'Sullivan B, Fornasier V, Wunder J, McLean M. Soft tissue sarcoma of the extremity. Limb salvage after failure of combined conservative therapy. Radiotherapy and oncology. 1996;41:209-14.

23. Torres MA, Ballo MT, Butler CE, Feig BW, Cormier JN, Lewis VO, et al. Management of locally recurrent soft-tissue sarcoma after prior surgery and radiation therapy. International Journal of Radiation Oncology* Biology* Physics. 2007;67:1124-9.

24. Kotilingam D, Lev DC, Lazar AJF, Pollock RE. Staging soft tissue sarcoma: evolution and change. CA: a cancer journal for clinicians. 2006;56:282-91.

25. Richardson ML, Zink-Brody GC, Patten RM, Koh W-J, Conrad EU. MR characterization of postirradiation soft tissue edema. Skeletal Radiol. 1996;25:537-43.

26. Bacci G, Lari S. Current treatment of high grade osteosarcoma of the extremity. Journal of chemotherapy. 2001;13:235-43.

27. Pennington Z, Ahmed AK, Cottrill E, Westbroek EM, Goodwin ML, Sciubba DM. Systematic review on the utility of magnetic resonance imaging for operative management and follow-up for primary sarcoma-lessons from extremity sarcomas. Annals of translational medicine. 2019;7.

28. Moulton JS, Blebea JS, Dunco DM, Braley SE, Bisset 3rd GS, Emery KH. MR imaging of soft-tissue masses: diagnostic efficacy and value of distinguishing between benign and malignant lesions. AJR. American journal of roentgenology. 1995;164:1191-9.

29. McKeon KE, Wright BT, Lee DH. Accuracy of MRI-based diagnoses for distal upper extremity soft tissue masses. Journal of hand and microsurgery. 2015;7:61-6.

30. Chung WJ, Chung HW, Shin MJ, Lee SH, Lee MH, Lee JS, et al. MRI to differentiate benign from malignant soft-tissue tumours of the extremities: a simplified systematic imaging approach using depth, size and heterogeneity of signal intensity. The British journal of radiology. 2012;85:e831-e836. 
31. Lin G, Yang L-Y, Huang Y-T, Ng K-K, Ng S-H, Ueng S-H, et al. Comparison of the diagnostic accuracy of contrast-enhanced MRI and diffusion-weighted MRI in the differentiation between uterine leiomyosarcoma/smooth muscle tumor with uncertain malignant potential and benign leiomyoma. Journal of Magnetic Resonance Imaging. 2016;43:333-42.

32. Fayad LM, Jacobs MA, Wang X, Carrino JA, Bluemke DA. Musculoskeletal tumors: how to use anatomic, functional, and metabolic MR techniques. Radiology. 2012;265:340-56.

33. Griffiths HJ, Thompson RC, Nitke SJ, Olson PN, Thielen KR, Amundson P. Use of MRI in evaluating postoperative changes in patients with bone and soft tissue tumors. Orthopedics. 1997;20:215-20.

34. Chou S-HS, Hippe DS, Lee AY, Scherer K, Porrino JA, Davidson DJ, et al. Gadolinium Contrast Enhancement Improves Confidence in Diagnosing Recurrent Soft Tissue Sarcoma by MRI. Acad Radiol. 2017;24:615-22. doi:10.1016/j.acra.2016.12.010.

35. Hirschmann A, van Praag VM, Haas RL, van de Sande, Michiel AJ, Bloem JL. Can we use MRI to detect clinically silent recurrent soft-tissue sarcoma? European radiology. 2020.

\section{Tables}

Due to technical limitations, table pptx are only available as a download in the Supplemental Files section.

\section{Figures}

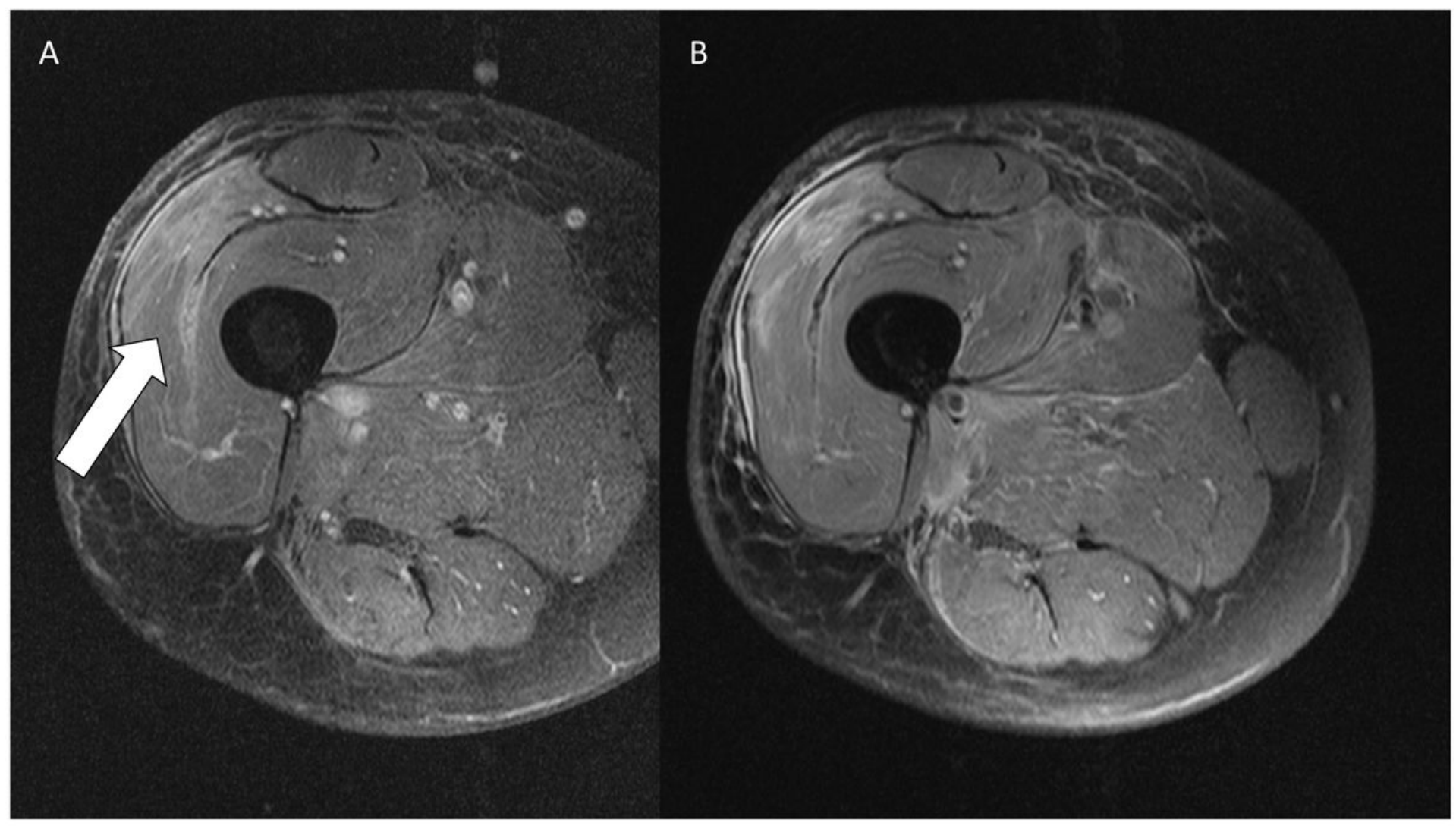




\section{Figure 1}

1.5-T MRI of the thigh (A: contrast-enhanced T1-weighted image with fat saturation in axial view; $B$ : Proton density-weighted image with fat saturation in axial view). Post-treatment muscle edema (white arrow) is shown without recurrent STS.

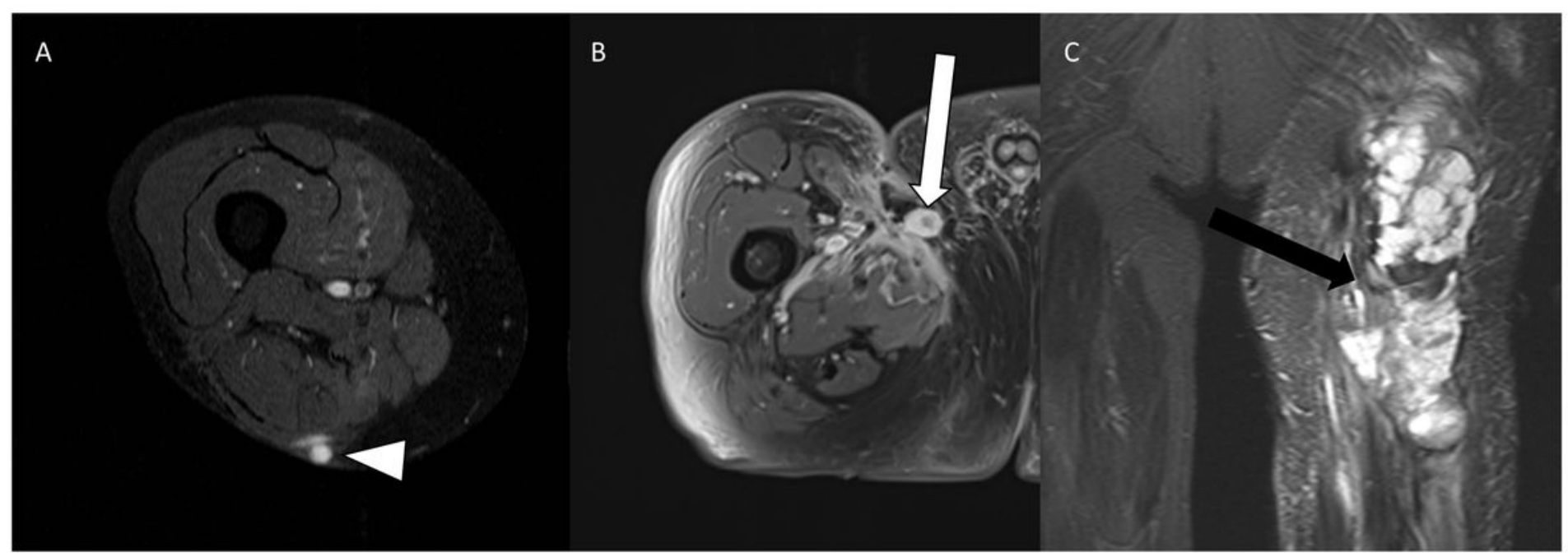

\section{Figure 2}

1.5-T MRI (A: contrast-enhanced T1-weighted image with fat suppression in axial view of the thigh; B: Contrast-enhanced T1-weighted image with fat suppression in axial view of the groin; C: Proton density weighted image with fat suppression in coronal view of the thigh). (A) shows a small nodular recurrence with slight edema (white arrowhead). (B) presents an ovoid/nodular recurrence (white arrow), which can easily be misinterpreted as a lymph node. (C) shows a multilobulated recurrence (black arrow) with adjacent edema.

\section{Supplementary Files}

This is a list of supplementary files associated with this preprint. Click to download.

- Table1.pptx

- Table2.pptx

- Table3.pptx

- Table4.pptx

- Table5.pptx 